\title{
Die Weiberherrschaft im Osmanischen Reich anhand von Johannes Tralows Roman: „Roxelane“.
}

$\underline{\text { Dr. Yasser Atef Abdullah Muhammad }}{ }^{(*)}$

\section{Einleitung}

Es gibt keinen Zweifel daran, dass die Literatur als ein Gesellschaftsspiegel gilt, der die Geschichte, die Zivilisationen und die Kulturen der Völker widerspiegelt. Daher variieren die Literaturen in verschiedenen Tendenzen je nach der Mannigfaltigkeit, der Vielfältigkeit der Kulturquellen und Ressourcen. Demzufolge unterscheiden sie sich von Nation zu Nation, von Kultur zu Kultur und von Zeit zu Zeit.

Wenn man die osmanische Geschichte betrachtet, findet man die Geschichtsschreiber des osmanischen Reiches weitgehend über die Frauen betreffenden Angelegenheiten zu schweigen. Auch Hofchroniken enthalten kaum Auskünfte über das Leben der Haremsbewohnerinnen. Die Abstammung der Konkubinen wurde historiographisch bewusst vernachlässigt. Die ursprünglichen Namen der Konkubinen sind in der Regel nicht bekannt, weil sie im Harem neue Namen erhielten. Der Harem blieb als Wohnort, Erziehungsanstalt und sozialer Raum lange Zeit geheimnisvoll und unerforscht.

\subsection{Auswahl des Gegenstandes}

Nachdem ich mich mit dem Roman „Mohammed“ von Tralow beschäftigt hatte, fand ich es sinnvoll und wichtig, Tralows berühmtestes Werk „Die osmanische Tetralogie“ zu behandeln. Aufgrund des Umfangs dieses Werkes habe ich

${ }^{(*}$ Dozent an der Philosophischen Fakultät der Sohag Universität. 
bevorzugt, den wichtigsten und bekanntesten unter den vier Romanen auszuwählen, und zwar „Roxelane“.

Die Kritiker und Leser bezeichnen die vier Bände ,Malchatun', ,Irene von Trapezunt', ,Roxelane', ,Der Eunuch' als das Kernstück der schriftstellerischen Werke Johannes Tralows. ${ }^{1}$ Sie gehören, vor allem ,Roxelane', zu den meistgelesenen und beliebtesten Büchern des Dichters, der sie als fast Sechzigjähriger $\mathrm{zu}$ schreiben begann und ihnen eineinhalb Jahrzehnte seines Lebens widmete, wie er selbst sagte,

„Als ich 1927 meinen Roman , König Neuhoff' so weit vorangetrieben hatte, bis Neuhoff als Gesandter nach Tunis sollte, erschrak ich plötzlich, weil mir bewusst wurde, dass ich von der türkischen Geschichte gerade in ihren wesentlichsten Teilen nicht genug wusste. Damals ließ ich mir zum ersten Mal den ,Hammer', die Geschichte des Türkischen Reiches, kommen. Wer dies mehrbändige Werk in die Hand nimmt, kann sich denken, dass mir dadurch eine sehr große Arbeit zufiel. [...] Mein Aufenthalt im Orient war mir natïrlich insofern förderlich, als ich mich in den Stoff leichter einfühlen konnte. Aber genaue Geschichtskenntnisse waren mir dadurch nicht vermittelt worden, sie galt es erst zu erwerben. [...] Nach 1945 reifte in mir der Gedanke von der Notwendigkeit, die Anfänge des Osmanischen Reiches - in dem Roxelane eine so bedeutende Rolle spielte - überzeugend zu schildern $[\ldots] . \omega^{2}$

${ }^{1}$ Stötzer, Helga: Johannes Tralow liest aus seinen Romanen Der Eunuch und Roxelane 1 Schallplatte Vinyl DDR Litera LP 860110.

2 Tralow, Johannes: Leben und Werk, (Hg) Deutsche Staatsbibliothek, Berlin. 1968, S. 29. 
Hier versenkt sich der Dichter in die Vergangenheit des Osmanischen Reiches von seiner Gründung bis zum langsamen Verfall, die er absolut tendenzfrei in Darstellung und Kommentierung zeichnet:

„weder den Eroberer, noch Süleyman als machtgierige, grausame Tyrannen, noch die osmanische Welt jemals als eine in Zeremonien erstarte Welt hingestellt oder von einem türkischen Feudalstaat gesprochen zu haben. "'l

Tralows Ziel war es, seinen Lesern geschichtliche Urteilsfähigkeit zu vermitteln, zugleich sollte jeder seiner historischen Romane ein Stück Gegenwartsliteratur sein. Tralow hat sich von Anfang an mit historisch weit zurückliegenden Themen beschäftigt. Das Geheimnis seines Erfolgs liegt vor allem darin, dass er für seine europäischen Leser unbekannte Welten öffnet.

Aus dieser Warte war meine Entscheidung, sich mit dem literarischen Werk „Roxelane“ von Johannes Tralow zu beschäftigen.

\subsection{Bedeutung des Werkes}

Tralows Roman „Roxelane“ gilt als das bekannteste und meistgelesene Werk in der osmanischen Tetralogie. Dieser Roman deckt die geheimnisvollen Konflikte und Ereignisse innerhalb des Harems auf.

\footnotetext{
${ }^{1}$ Kaufmann, Max R.: Osmanische Trilogie. Die historischen Romane von Johannes Tralow. In: Mitteilungen der Deutsch-Türkischen Gesellschaft e.V. Bonn, Nr. 10 vom April 1956, S. 10f.
} 
Nachdem Suleiman der Prächtige 1520 den Thron bestiegen hatte, gelangten die Frauen der osmanischen Dynastie in eine Machtposition, die in der osmanischen Geschichte einmalig war. Diese Epoche wird als „Weiberherrschaft" ${ }^{\text {"1 }}$ bezeichnet.

Tralow schildert sachkundig und fassettenreich die türkische Geschichte und die Weiberherrschaft im osmanischen Reich, die in der deutschen Literatur weitgehend nicht behandelt werden.

Es wird davon deutlich, dass Tralow die türkische Geschichte als einen Teil der europäischen Geschichte auf einfache Weise durch „Die osmanische Tetralogie“ darstellen und es beliebter und interessanter machen will. Über seine Absicht sagt Tralow:

„Ich hoffe, mit meiner ,Osmanischen Tetralogie' es meinen Lesern möglich gemacht zu haben, die türkische Geschichte - vom 13. bis zum 17. Jahrhundert - als einen Teil der europäischen zu begreifen. “2

In diesem Zyklus stellt Tralow den Aufstieg der Osmanen vom türkischen Nomaden-Stamm zur Weltmacht sowie den beginnenden Zerfall (13. bis 18. Jahrhundert) dar.

${ }^{1}$ Peirce, Leslie (1992): Beyond Harem Walls: Ottoman Royal Women and the Exercise of Power. In: Helly, D. O./Reverby, S. M. (Hrsg.) (1992): Gendered Domains. Rethinking Public and Private in Women's History. London, S. 41.

${ }^{2}$ Kaufmann (1956), S. 29. 


\subsection{Ziele der Untersuchung.}

Ziel der Arbeit ist es, die Weiberherrschaft im osmanischen Reich durch Tralows Roman „Roxelane“ aufzuzeigen sowie die verborgenen Konflikte und Ereignisse innerhalb des Harems aufzudecken. Auch erklärt diese Arbeit den großen Einfluss der Haremsfrauen auf den mächtigen Sultan Suleiman.

Die Arbeit stellt viele problematische Fragen, die im Rahmen dieser wissenschaftlichen Forschung nach passenden Antworten suchen.

1- Wer ist Tralow?

2- Was versteht man unter dem Begriff „Harem“?

3- Was versteht man unter dem Begriff „Weiberherrschaft"?

4- Wie war das Bild von Roxelane in der deutschen Literatur?

5- Wie hat Roxelane die osmanischen Traditionen beseitigt?

6- Welche Rolle spielte Roxelane zum Schutz ihrer Kinder vor Mord?

7- Was ist die diplomatische Rolle von Roxelane gegenüber den anderen Staaten?

8- Was sind die islamischen Fragen, die Tralow im Roman behandelt?

9- Inwieweit ist es ihm gelungen, die verborgenen Ereignisse des osmanischen Reiches darzustellen?

\subsection{Hypothesen der Untersuchung.}

In dieser Arbeit werden einige Hypothesen vorausgesetzt, die im Laufe der Zeit mit schlagenden Argumenten zu beweisen sind. 
1- Roxelane war die wahre Partnerin Suleimans in der Herrschaft des Osmanischen Reiches.

2- Sie konnte im Laufe der Zeit die strengen Gesetze und Traditionen im Osmanischen Reich verändern.

3- Ihre Verschwörungen und Auseinandersetzung mit anderen Frauen im Harem hatte das Ziel, ihre Kinder vor Mord zu schützen.

4- Rechtfertigung des Autors für Roxelanes Taten und dessen Verteidigung sie.

5- Tralow hat einige islamische Fragen aufgeworfen.

Bevor wir uns mit dem Roman „Roxelane“ beschäftigen, wollen wir kurz auf einige Aspekte eingehen:

\section{Überblick über den Autor Johannes Tralow $^{1}$}

Johannes Tralow wurde im Jahre 1882 in Lübeck als Sohn eines Kaufmanns geboren. Nach dem frühen Tod des Vaters blieb die Mutter alleine und der kleine Johannes war 5 Jahre alt. Er half seiner Mutter im Geschäft. Tralow besuchte die private Großheimsche Realschule. Nach dem Schlussexamen der Realschule kam er ins Realgymnasium, ins Katharineum, zu dessen Schülern auch Theodor Storm sowie Heinrich und Thomas Mann zählen. ${ }^{2}$

${ }^{1}$ Es ist bemerkenswert, dass es kein vollständig wissenschaftliches Werk über Tralow-Biographie bisher gibt. Informationen über sein Leben findet man in seiner autobiographische Skizze „Der Anfang“ von 1958, sowie einer Broschüre „Johannes Tralow - Leben und Werk“, die seine letzte Lebensgefährtin Helga Stötzer 1968 für die Deutsche Staatsbibliothek Berlin zusammengestellt hat.

2 Tralow, Johannes: Leben und Werk, (Hg) Deutsche Staatsbibliothek, Berlin. 1968. S. 7. Und Vgl. Johannes Tralow 1882-1968: 
Wegen der Ausbreitung von Krankheiten in der Schule litt Tralow an Tuberkuloseverdacht. Deshalb brauchte er heißes Klima. So ersparte die Mutter etwas Geld und verschickte den 17jährigen nach Ägypten, um durch das heilkräftige Wüstenklima Ägyptens von einer Lungenerkrankung zu genesen. ${ }^{1}$

Nach der Rückkehr begann er zunächst eine kaufmännische Laufbahn wie seine Eltern, daneben arbeitete er als Journalist beim liberalen „Lübecker Tagblatt“،, zu dessen Chefredakteur er aufstieg. ${ }^{2}$

1907 wurde Tralow sein Versdrama „Das Gastmahl zu Pavia“ aufgeführt. Es folgten weitere Theater-arbeiten. 1910 bis 1914 leitete er den Berliner Theaterverlag und erwarb er sich einen Namen als Regisseur und Theaterleiter in Halle, Mainz, Nürnberg, Berlin, Köln, Kassel, Hamburg, u.a. Städte.

1922 nannte ihn Carl Steinheim als einen der drei bedeutendsten Regisseure Deutschlands. ${ }^{3}$ Danach arbeitete er als freier Schriftsteller überwiegend an seinen historischen Romanen, durch die er überall bekannt wurde. 1945 wurde er Oberster Richter der ersten Spruchkammer in Starnberg zur

(http://www.katharineum.de/rundgang/beruehmte/rundgang/beruehmte /johannes-tralow-1882-1968) [stand: 5.10.2011].

1 Tralow: Leben und Werk, a.a.O., S. 15.

2 Vgl. Johannes Tralow, Ein Meister des historischen Romans. In: Mitteilungen der Deutsch-Türkischen Gesellschaft, 76, Bonn 1968, S. 11.

${ }^{3}$ Ebenda, S. 11 
Untersuchung nazistischer Tätigkeiten und leitete diese bis $1947 .{ }^{1}$

Seit 1929 war Tralow Mitglied der internationalen Schriftstellervereinigung P.E.N. Nach dem Krieg wurde er 1948 in deren Vorstand gewählt. Wegen der Zunahme der ostdeutschen Autoren im P.E.N. entstand 1951 eine westdeutsche Gegengründung. Tralow wurde dabei zum Präsidenten des deutschen P.E.N.-Zentrums gewählt. ${ }^{2}$

Schließlich übersiedelte er in die DDR, der er sich geistig zugehörig fühlte. Am 27. Februar 1968 starb Johannes Tralow in Berlin. ${ }^{3}$

Im Januar 1972 widmet ihm die DDR-Post anlässlich seines 90sten Geburtstags eine Briefmarke der Serie „Berühmte Persönlichkeiten“.

\section{Der „Harem“ als Machtzentrum}

Der Ausdruck Harem wird vom Wort „harim“ (harīm) abgeleitet, der sowohl ,verboten', tabu' als auch ,heilige' (unverletzlicher Ort, geheiligter Bereich) bedeutet. Er bezeichnet einen abgeschlossenen und bewachten Wohnbereich eines Serails. Auch die für Nichtmuslime

1 Anmerkungen zu einer Biographie, Johannes Tralow in die DDR übergesiedelt, In: Deutsche Fragen. Informationen und Berichte, Berlin, S. $171 \mathrm{f}$.

2 Meyers Neues Lexikon, 2. Aufl., Leipzig 1976, Bd. 13, S. 665.

${ }^{3}$ Johannes Tralow, Der Nachlass, Hrsg. H. Stötzer, H. Döhn, Berlin 1977, Deutsche Staatsbibliothek, S.V. 
verbotenen Areale in Mekka und Medina tragen die Bezeichnung Harām. ${ }^{1}$

Die osmanischen Sultane waren eigentlich keine sakralen Könige, schufen aber durch ihre Anwesenheit sakrale Orte. Bis zum Ende des 16. Jahrhunderts bezeichnete das Wort „Harem“ noch das Innere des Hofes, in dem der Sultan mit anderen Männern zusammen wohnte. Im Osmanischen Reich repräsentierte das „Innen“, der Wohnraum des Sultans, Ordnung, Sicherheit und das Zentrum der politischen Herrschaft. $^{2}$

In vielen absoluten Monarchien galt die Nähe zur Person des Königs als eine Machtquelle. Die Unerreichbarkeit des Herrschers verlieh ihm eine sakrale Aura, an der nur die ihm räumlich nahe stehenden Personen partizipierten.

Nachdem die Osmanen die Reste des byzantinischen Reiches erobert hatten, machten sie Konstantinopel unter dem Namen Istanbul zur Hauptstadt. Mehmet II. (der Eroberer) zog dort in einen neu errichteten und heute unter anderem für seine Haremsräume berühmten Palast, Topkapi. Der Topkapi-Serail war dagegen jahrzehntelang nur Eunuchen und Knaben in der Ausbildung vorbehalten. Als ein Teil der Frauen mit ihren Kindern - während der Herrschaftszeit Suleimans - in den

${ }^{1}$ Leslie P. Peirce: The imperial harem: women and sovereignty in the Ottoman Empire. Oxford University Press, New York 1993, S. 3-5.

2 Andrews, Walter: Poetry's Voice, Society's. 1985, Seattle. In Kürsat, Elçin: Haremsfrauen und Herrschaft im Osmanischen Reich. In: Zeitschrift: Nachdenken über..., Stuttgart, 2003, Band: 21, Heft: 1, S. 36. 
Topkapi umzogen, trug dies zum symbolischen und realen Machtanstieg der Frauen bei. $\mathrm{Zu}$ diesem Zeitpunkt wurde im Topkapi ein neuer Wohnbereich gebaut, der - wegen der Anwesenheit des Sultans „Harem“ genannt - sich zur prestigereichen Wohnstätte der höfischen Frauen entwickeln

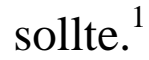

Im Harem bildeten die Mütter mit den Prinzen eine symbiotische Einheit. Sie waren Beschützerinnen gegen Intrigen und gegen den Zorn des Sultans, Erzieherinnen, später Beraterinnen und engste Lebensgefährtinnen des Prinzen. Das Schicksal und die Aufstiegschancen der Mütter waren mit ihren Kindern verbunden. In einer Herrschaftsordnung, in der jeder Prinz mit seinen Brüdern von unterschiedlichen Müttern um den Thron konkurrierte, bedurfte jeder potenzielle Thronkandidat der Obhut einer ihm wohlgesonnenen Mutter. Die Konkurrenz um die höchste Spitze im Reich, um das Sultanat, war gleichzeitig eine Konkurrenz unter den Müttern. Denn sie waren diejenigen, die diesen Konkurrenzkampf durch ihr Vermögen, ihre Hofbeziehungen und Klientelgruppen organisierten und ausfochten.

Die Konkurrenz um den Thron konnte nur siegreich verlaufen, wenn die Unterstützung wichtiger Machtgruppen, wie der Janitscharen, der hochrangigen Staatsfunktionäre und islamischen Rechtsgelehrten, gesichert war. ${ }^{2}$

\footnotetext{
${ }^{1}$ Kürsat (2003), S. 36.

${ }^{2}$ Ebenda, S. 39.
} 


\section{,Weiberherrschaft ${ }^{66}$ in der Osmanischen Geschichte}

„Weiberherrschaft" bezeichnet einen Zeitraum in der osmanischen Geschichte, in dem Frauen bedeutenden Einfluss auf die Politik des Osmanischen Reiches nahmen. Der Begriff wurde zumeist benutzt, um den inneren und äußeren Niedergang des Osmanischen Reiches seit dem 16. Jahrhundert zu erklären.

Hurrem, die als „Roxelane“, die Lieblingsfrau Suleimans des Prächtigen, bezeichnet wurde, bestimmte nicht nur den Beginn dieser sogenannten „Weiberherrschaft", sondern galt auch als die bekannteste Persönlichkeit in diesem Zeitraum. Mit ihr führte Suleiman lebenslang eine monogame Beziehung. Sie war die erste Haremsfrau, die einen besonderen Titel und Status einer „Haseki“" gegenüber anderen Konkubinen erhielt.

Polnischen Berichten zufolge war Hurrem - in Europa unter dem Namen Roxelane bekannt- in der Stadt Rogahin am Dnjestr von Tataren gefangen genommen worden. Sie hieß ursprünglich Aleksandra Lisowaka und war die Tochter eines christlichen Geistlichen. Wie sie in den Sultansharem gelangte, ist ungewiss. ${ }^{1}$

Durch Tralows Roman „Roxelane“ werde ich Einzelheiten ihres Lebens und ihren Einfluss auf Suleiman darstellen.

\section{Tralows Roman ,Roxelane“6.}

„Jeder historische Roman ist Gegenwartsliteratur, sofern der Autor nur gegenwärtig denkt, handelt und fühlt. “2

\footnotetext{
${ }^{1}$ Meram, Ali Kemal: Padisah Analari. Istanbul, 1997. Zitiert nach Kürsat (2003), S. 39.

2 Tralow: Leben und Werk. a.a.O., S. 26.
} 
Mit diesem Satz sagte „der Meister des Historischen Romans" Johannes Tralow das Wesentlichste über sein Gesamtwerk. Die Betrachtungen von Tralow zu den einzelnen Romanen geben Aufschluss über ihr Entstehen und das Anliegen des Schriftstellers.

Tralows Roman „Roxelane“ ist der dritte Band der Osmanischen Tetralogie. Wie in den ersten zwei Bänden der Tetralogie ist wieder eine Frau die Titelheldin, die abermals maßgeblichen Einfluss auf die Ereignisse um das Osmanische Herrscherhaus hat.

Dieser Roman spielt zur Blütezeit des Osmanischen Reiches unter der Herrschaft des osmanischen Sultans Suleiman I., der der mächtigste und von den christlichen Herrschern gefürchtetste Mann des 16. Jhs. war. Er wurde im Jahre 1494 als Sohn Selims I. „des Grausamen“ und dessen schönen Hauptkonkubine Walideh in Trapezunt am Schwarzen Meer geboren. ${ }^{1}$ Nach dem Tod seines Vaters im Jahre 1520 trat er dessen Nachfolge an und sorgte durch zahlreiche Kriege dafür, dass das osmanische Reich die größte Ausdehnung und die größte Machtentfaltung in der türkischen Geschichte unter seiner Herrschaft erreichte. Es erstreckte sich von Ungarn bis in den Jemen, von Westpersien bis nach Nordafrika und war von einer Reihe von Satellitenstaaten umgeben.

${ }^{1}$ Suleiman I. wurde von Europäern „der Prächtige“ und von Türken „der Gesetzgeber" (Qānūni) genannt. Denn er ist der Schöpfer der Osmanischen Reichesverfassung und Former des türkisch-islamischen Rechts, das für Jahrhunderte Gültigkeit haben sollte.

Vgl. Schreiber, Hermann(Hrsg): Weltreiche(2)- Das Imperium Romanum.

Das Heilige Römische Reich. Byzanz. Die Osmanen, S. 273. 
Seine große Liebe zu Rosska, dem elternlosen Tatarenmädchen, macht aus ihr ,Roxelane', die Lieblingsfrau Sultan Suleimans I. und schließlich sogar Kaiserin. Dieser außergewöhnliche Aufstieg erfüllte sich durch ihre eigenartige tatarische Erscheinung und ihre Klugheit. Ihre Liebe zu ihrem Mann und zu ihren Söhnen wie ihr unermüdlicher Kampf gegen die Haremscliquen bestimmen den Inhalt dieses Romans.

Roxelane wurde von Ibrahim Pascha, dem Freund Suleimans, gekauft, der selbst in jungen Jahren als griechischer Gefangener Suleiman I. geschenkt worden war. Er galt als hochbegabt, bezaubernd, arrogant, energisch, zielbewusst, konnte sich in mehreren Sprachen fließend unterhalten, spielte Geige und schrieb Lieder und Gedichte.

Suleiman, der sich in seiner Kindheit oft sehr einsam gefühlt hatte, schätzte seinen Sklaven Ibrahim Pascha besonders wegen dessen Heiterkeit und dessen Witz. Ibrahim konnte dadurch den oft in schwere Depressionen fallenden Sultan wieder aufrichten. Später ernannte ihn Suleiman deshalb $\mathrm{zu}$ seinem Kammerherrn und ließ für ihn außerdem einen prächtigen Palast bauen.

Als Geschenk erhielt der Sultan von seiner Tante Nur Banu die junge Russin Roxelane überreicht ${ }^{1}$. Roxelane ist zierlich, schlank, anmutig und liebreizend und hatte auch flammend rotes Haar. Zudem war sie sehr intelligent und sehr lebhaft und

Vgl. Tralow, Johannes: Roxelane, Verlag der Nation, 13. Auflage, Husum, 2008, S. 58. 
lachte und scherzte gern. Wie alle anderen jungen Haremsdamen erhielt sie zuerst gründlichen Unterricht in der türkischen Sprache und im Islam. Ferner stand noch Schreiben, Lesen, das Abfassen von zierlichen Liebesbriefen, Nähen, Sticken, Singen, Tanzen, das Erlernen eines Musikinstrumentes und die Einübung von anmutigen und graziösen Bewegungen auf ihrem Stundenplan.
„Die Sultaninn Chasseki, die Russinn Churrem, [...] welche durch Reitz und Talent sich von der Sklavin nicht nur zur Gemahlin Suleiman's aufschwang, sondern als alleinige Genossin seines Bettes denselben auch, als schon lange die Reize ihrer Schönheit verblüht sein mussten, durch die Überlegenheit ihres Geistes und Charakters nach ihrem Willen lenkte [...] an der Seite des größten Kaisers der Osmanen, den das Weib, wie er das Reich, unumschränkt beherrschte".'

Die ungewöhnlich schönen und begabten Sklavinnen hatten die Chance Konkubinen des Sultans zu werden. Die 12 der attraktivsten und talentiertesten unter ihnen hatten Aufgabe, ihren Herrn anzukleiden, zu baden und mit köstlichen Mahlzeiten zu versorgen. Als mögliche Bettgenossinnen des Sultans konnten sie schließlich $\mathrm{zu}$ seinen Favoritinnen, den Ikbals, aufsteigen. Wurden diese schwanger, und brachten sie ihrem Herrn Kinder auf die Welt, erhielten sie den Ehrentitel Konkubinen oder Kadine des Sultans.

Für Roxelane war es nicht schwer, Suleimans Aufmerksamkeit zu erwecken. Außerdem zog ihr herzliches

${ }^{1}$ Hammer-Purgstall (Freiherr von), Joseph, Geschichte des osmanischen Reiches, Bd. 3: Vom Regierungsantritte Suleiman des Ersten bis zum Tode Selim's II. 1520-1574, Pest 1828, S. 350. 
Lachen jeden im Harem an. Deshalb erhielt sie schon bald den Beinamen „die Lachende“ oder „Khurrem“. Und so dauerte es auch nicht lange, bis sie zur Bettgenossin des Sultans bestimmt wurde. Suleiman war für sie begeistert, weil sie, nicht wie seine anderen Favoritinnen, nur zur Triebbefriedigung und Gebärerin, sondern auch als Gesprächspartnerin, als wahre Freundin und als Gefährtin fürs Leben war.

Mit Roxelane konnte er Gedichte austauschen oder politische Diskussionen führen. Sie war in der Lage, ihm kluge Ratschläge zu geben und hatte Verständnis für seine Ängste, die er nur ihr zu beichten wagte. Außerdem war sie fähig, mit ihrer Heiterkeit wie sein Freund Ibrahim seine Melancholie zu vertreiben. Immer häufiger suchte er schließlich nur noch sie auf und vernachlässigte seine Hauptfrau, die Montenegrinerin oder Tscherkessin Gulbehar, die den Beinamen „die Frühlingsblüte" führte und die ihm vier Kinder geboren hatte, von denen jedoch nur noch der Sohn Mustafa am Leben war.

Roxelane muss den Kampf mit dem Qānūn „Gesetz“ des Eroberers ausfechten, der bestimmt, dass der Thronerbe seine Brüder umbringen lassen kann, damit die Ruhe des Reiches gesichert bleibt. Um das Reich nicht nach dem Tode jedes Sultans einem Bürgerkrieg auszusetzen, weil dessen Söhne um die Nachfolge blutige Kämpfe ausführten, hatte Muhammad II. (1481) folgendes Gesetz erlassen:

„Wer von meinen erlauchten Söhnen und Enkeln zur höchsten Macht gelangt, der darf seine Brüder sterben lassen für die Ruhe der Welt. "W

${ }^{1}$ Tralow, Johannes: Roxelane, a.a.O., S. 51f. 
Dieses Dürfen war ein Muss; denn das Gesetz war hart, aber berechtigt wegen vieler Putschversuche. Seit diesem Erlass wiederholte sich nun anlässlich jedes Thronwechsels das gleiche grausame Ritual. Der neue Herrscher ließ seine sämtlichen Brüder und Halbbrüder und deren Söhne erdrosseln. Suleiman hat dieses Gesetz nicht ausgeführt, da sein Vater nicht nur seine eigenen zwei Brüder, sondern auch seine sieben Neffen und Suleimans vier Brüder erdrosseln ließ, um seinem Lieblingssohn die Thronfolge zu sichern. Aus diesem Grund wollte Roxelane keine Söhne von Suleiman.

„Mit ihren siebzehn Jahren wusste sie nichts von des Weibes schwerster Not, doch das wusste sie, dass sie nicht die Mutter kaiserlicher Knaben werden wollte, die nur heranwachsen würden, um einmal erwürgt zu werden. "l

Aber Roxelane stand trotz allem wie eine gebuckelte, fauchende Katze vor Suleiman

„Willst du dich gegen den Kanun des Eroberers auflehnen?

Ich will keine Söhne von dir! Keine Söhne von mir?! empörte sich Suleiman [... $]^{\mathrm{c}^{2}}$

Gegen ihren Willen schenkt sie dem Sultan Suleiman ihren ersten Sohn Muhammad, dann den buckligen Giangir - dessen Rücken Verwachsungen aufwies und deshalb er keine Ansprüche auf das Sultanat erheben konnte - Selim und Bajazet sowie die Tochter Mirmah. Roxelane gerät in Konflikt

Tralow, Johannes: Roxelane, a.a.O., S. 76.

2 Ebenda, S. 94. 
mit der Sultanin ,Saffiya', die dem Sultan bereits den Thronerben Mustafa geschenkt hat. Selbst die Sultansmutter, ihre bisherige Feindin, versöhnt sich mit Roxelane, doch sie weiß, dass diese als Mutter um das Schicksal ihrer Söhne kämpfen muss. Sie lässt Roxelane einen Eid schwören, dass sie alles tun werde, um das Gesetz des Eroberers nicht umzustoßen, allerdings auch dafür zu sorgen, dass Mustafa kein Leid widerfahre. Und damit begann für die junge Mutter das große Drama in ihrem Leben.

Roxelane selbst spielte in der Zwischenzeit im Leben ihres Mannes eine bedeutendere Rolle. Suleiman liebte seine flinke, heitere, zweite Hauptfrau über alles und suchte fast nur noch ihre Gesellschaft auf. Selbst seinen Freund Ibrahim Pascha begann er zu vernachlässigen, der zum Großwesir und zum Großgouverneur des Balkans aufgestiegen und mit einer Schwester Suleimans, Hatice, verheiratet worden war.

Roxelane beutete Sultans Liebe $\mathrm{zu}$ ihr aus, um ihre Wünsche zu erfüllen. Sie wollte Gulbehar, die erste Frau des Sultans, entfernen, so entwarf sie einen handfesten Streit mit ihr auszubrechen, bei dem die erste Kadin, Gulbehar, ihre Rivalin, Roxelane, heftig an den Haaren gezogen und deren Gesicht zerkratzt hatte. ${ }^{1}$ Suleiman verbannte daraufhin seinen ältesten Sohn Mustafa als Statthalter nach Manisa, einer Stadt in der westlichen Türkei, die weit genug vom Zentrum der Macht entfernt war. Seine Mutter, Gulbehar, musste ihn entsprechend dem osmanischen Protokoll dorthin begleiten.

${ }^{1}$ Vgl. Tralow, Johannes: Roxelane, a.a.O., S. 148. 
Damit gelang es Roxelane, sie loszuwerden und wurde die erste Frau des Sultans, insbesondere nach dem Sterben der Sultansmutter.

Fortan hatte Suleiman nur noch Geschlechtsverkehr mit Roxelane. Seine anderen schönen Sklavinnen vermählte er im Laufe der nächsten Jahre mit seinen Paschas. Zudem versuchte er alle Wünsche seiner geliebten Roxelane zu erfüllen und fand sich sogar bereit, sie zu ehelichen, was schon seit ungefähr 130 Jahren nicht mehr Sitte unter den osmanischen Sultanen war. ${ }^{1}$ Trotzdem fand die prachtvolle Hochzeit statt.

„Er [Suleiman] liebt sie so und hält ihr so sehr die Treue, dass sich alle seine Untertanen wundern und sagen, sie habe ihn verhext. Sie nennen sie die »Ziadi« oder die Hexe. Deshalb wird sie samt ihren Kindern vom Hof und vom Heer gehasst, aber niemand wagt etwas gegen sie zu sagen, weil er sie liebt. “2

${ }^{1}$ Nach der Niederlage von Bajazet (1402) gegen Timurlenk wurde seine Frau von Timurlenk, dem Sieger, zum Dienst in dessen Siegsfeier völlig nackt erzwungen, infolgedessen starb Bajazet vor Trauer und Wut. Nach diesem Ereignis wurde die Ehe verboten. Vgl. Tralow, Johannes: Roxelane, a.a.O., S. 164 und Al-Serganyī, Raġīb: AlMawsū't al Mūyasāratī fi a Tarìg al Islamī (Die erleichterte Enzyklopädie in die islamische Geschichte), 7. Auflage, Mū'sasat Ikr' lil nashr wa tāwz wa tarğma Verlag, Kairo, 2007. S. 130.

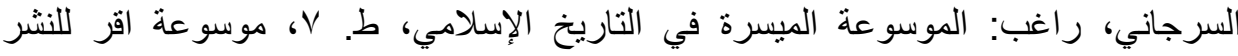

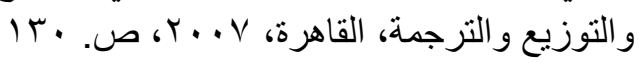

2 Barber, Noel: Die Sultane - Die Geschichte des Ottomanischen Reiches dargestellt in Lebensbildern, Frankfurt a.M., Berlin, Wien 1975², S. $33 \mathrm{f}$. 
Voller Neid verfolgten die Untertanen die große Liebe ihres Herrn zu dieser ehemaligen Sklavin, deren Einfluss am Hofe ihres Gatten im Laufe der Jahre weiter zunahm.

Als Suleimans einziger Freund und Großwesir Ibrahim Pascha seinem Herrn über den Kopf zu wachsen schien und bedeutender als der Sultan selbst zu sein, wurde er bereit für den Tod. Hatte Ibrahim Pascha doch folgendes verkünden lassen:

„Es ist wahr, dass ich dieses große Reich regiere [...] Was ich auch anfasse, es wird durchgeführt. Wenn ich wollte, könnte ich aus einem Stallburschen einen Pascha machen. Was ich zu geben wïnsche, wird gegeben und kann nicht genommen werden. Mein Herr wird nichts dagegen einwenden. Wenn der große Sultan etwas gibt, und ich bin nicht damit einverstanden, dann wird es genommen. Die Entscheidung über Krieg und Frieden, die Verwendung der Staatsmittel - das alles liegt in meiner Hand. Der Sultan ist nicht besser gekleidet als ich. Seine Vollmachten vertraut er meinen Händen an $[\ldots]^{\text {'ll }}$

Wer solches auszusprechen wagte, würde auch nicht zögern, einen Umsturz $\mathrm{zu}$ initiieren. Und vor nichts hatte Suleiman solche Angst wie vor einer Rebellion, die seine Absetzung beinhalten konnte.

Aber der Sultan hat einen Eid geschworen, seinem Freund niemals ein Leid anzutun, und von diesem Eid muss der Sultan entbunden werden. Aber Ibrahim soll für seine Schandtaten ${ }^{2}$

Lamb, Harold: Suleiman der Prächtige, München 1952 S. 162.

2 Vgl. Tralow, Johannes: Roxelane, a.a.O., S. 402 „In seinem Haus hat Ibrahim dir Schande angetan.[...] Du sollst wissen, dass Ibrahim nach 
bestraft werden. Das Fatwa, das Roxelane vom Mufti erwirkt, lautet:

„Es steht geschrieben: Der Schlaf gleicht dem Tode, also ist der Schlafende nicht als Lebender zu achten "I

So kann Ibrahim erdrosselt werden, ohne dass der Sultan seinen Eid bricht.

Nach dem Tode Ibrahim Paschas blieb dem Sultan nur noch Roxelane als einzige Freundin, Ratgeberin und Lebensgefährtin, bei der er sein Herrscheramt ablegen konnte und ein gewöhnlicher Mensch mit all seinen Schwächen sein durfte.

1541 rückten die beiden Ehegatten auch örtlich näher zusammen. Denn ein Großbrand hatte im Eski-Sarayi, dem Palast des Harems, einen Großteil der Gebäude in Schutt und Asche gelegt. Roxelane konnte ihren Gatten überreden, den Palast nicht wieder aufzubauen, sondern ihr Domizil in Zukunft in seine Nähe zu verlegen, damit die Entfernung zwischen ihnen nicht mehr so groß sei. Suleiman willigte sofort ein und ließ auf dem Areal des Topkapi Sarayi die Gebäude des neuen Harems errichten. Da er mittlerweile schon sehr unter der Gicht litt, konnte er somit auch schneller und länger unter der Obhut seiner Gattin gepflegt und aufgeheitert werden.

Im Jahre 1543 verlor Roxelane ihren ersten Sohn Muhammad, der beim Volk und beim Heer sehr beliebt war

der Scham deines Weibes griff. Wirst du ihn auch dafür ungestraft lassen?"

1 Tralow, Johannes: Roxelane, a.a.O., S. 407. 
und der seinem Vater als Sultan folgen sollte. Er wurde jedoch nur 21 Jahre alt und hinterließ nur eine zweijährige Tochter. Nach seinem Tod brach bei Roxelane die alte Angst wieder auf, dass ihr Stiefsohn Mustafa, den sie zu schützen gelobt hat, ihre übrigen drei Söhne nach dem Qānūn (Gesetz) ermorden würde. Sie verfällt der Schwermut, bleibt aber mächtig als Beherrscherin der kaiserlichen Umgebung, und ihre Macht wächst sich mit der Ernennung ihres Schwiegersohns Rustem, des Mannes ihrer Tochter Mirmah, zum Großwesir. Schwermut und Angst treiben sie $\mathrm{zu}$ theosophischen Betrachtungen und Grübeleien.

Mit dem Gatten ihrer Tochter Mirmah, Rustem Pascha versuchte sie in den folgenden Jahren deshalb alles, um Mustafa als potentielle Gefahr für ihre Kinder auszuschalten.

Um den Sultan zur Tötung seines Erstgeborenen Mustafa $\mathrm{zu}$ treiben, verbreitete sich ein Gerücht ${ }^{1}$, dass Suleimans ältester Sohn einen Umsturz gegen seinen Vater plant. Roxelane als Mutter ihrer vom Gesetz (Qānūn) bedrohten Söhne unternahm - trotz des geschworenen Eides - nichts zur Rettung ihres Stiefsohnes. Suleiman hielt jedenfalls die Informationen über die Rebellion seines Sohnes für wahrhaftig. Deshalb ließ er ihn und dessen 11-jährigen Sohn, seinen eigenen Enkel, als Hochverräter mit einer seidenen Bogensehne erdrosseln. ${ }^{2}$

1 Historisch ist nicht zu verifizieren, ob dieses Gerücht richtig ist, oder ob dies alles nur von Roxelane und ihrem Schwiegersohn Rüstem Pascha gerüchtmäßig verbreitet wurde.

${ }^{2}$ Vgl. Tralow, Johannes: Roxelane, a.a.O., S. 517. 
Da traten sich Unruhen zwischen den Janitscharen ${ }^{1}$ im Heer auf, bei denen Mustafa sehr beliebt war. Sie wurden für den Sultan so bedrohlich, dass er auf ihren Druck seinen Schwiegersohn, den Großwesir Rustem Pascha, seines Amtes entheben musste. Er wurde überall als Drahtzieher des Komplotts gegen Mustafa angesehen.

Nachdem der Aufstand unter den Janitscharen durch üppige Handgelder besänftigt werden konnte und sich die Wogen wieder geglättet hatten, wurde Suleimans Schwiegersohn 1555 erneut zum Großwesir ernannt.

Natürlich wurde Roxelane bei all diesen Mordtaten - auch bei der Hinrichtung Ibrahim Paschas - stets als treibende Kraft gesehen, was jedoch quellenmäßig nicht im Geringsten bewiesen werden kann. Andererseits hätte wohl kaum eine Mutter die bevorstehende Ermordung ihrer Söhne hingenommen, ohne alles zu versuchen, um dies zu verhindern. Suleiman kränkelte schon seit mehreren Jahren. Mit seinem Tod musste Roxelane jederzeit rechnen. Als Kämpferin von Natur aus konnte und wollte sie ihre Söhne dem Henker nicht so einfach übergeben.

Die Welt aber kennt ihre Gewissenskonflikte nicht, nicht die Beweggründe, die sie die Beseitigung Ibrahims

${ }^{1}$ Die Janitscharen bestand aus jugendlichen Kriegsgefangenen und Knaben der unterworfenen christlichen Völker, die ihren Eltern weggenommen, zum Islam bekehrt, in besonderen Schulen hart ausgebildet und bedingungslos auf Treue zum Sultan verpflichtet wurden. Sie blieben auch nach ihrer Ausbildung kaserniert und unterlagen einer harten Zucht. So war es ihnen verboten einen anderen Beruf auszuiuben und zu heiraten. Vgl. Tralow, Johannes: Roxelane, a.a.O., S. 97. 
befürworten ließen, und so geht ihr Name als der einer Teufelin durch die Welt. Ihre Macht steigt, aber seelisch ist sie geschlagen. Ist sie schuldig geworden?

Der Schriftsteller Tralow hat sich in ihrer Seele versenkt und lässt ihr durch den Popen in Choritza, ihrem Heimatort, sagen:

„Gott ist die Liebe und sie ist unteilbar, wie Gott selbst. Dass du nicht, wie Er, zu lieben vermagst-dafür bist du ein Mensch. Aber, wer viel liebt, dem wird vergeben. "'

So lässt Roxelane ihre Moschee und ein Armenhaus für Frauen anbauen, für ihren Giangir eine Moschee erstellen und weiht dem Andenken ihrer Tochter Mirmah die Moschee in Üsküdar. Suleiman aber wird die Sulaimaniye-Moschee bauen lassen. Mit dem Sultan wird Roxelane die Macht des mächtigsten Herrschers der Welt teilen.

Während der Herrschaft Sulaimans gab es einen aktiven diplomatischen Austausch zwischen Roxelane und anderen europäischen Dynastien. Die Beteiligung der dynastischen Frauen an der Diplomatie hatte in den Turkstaaten eine lange geschichtliche Tradition. Kennzeichnend für die Beziehungen war, dass die Frauen sich auf ihre Herkunftsstaaten orientierten und sich ihnen gegenüber als parteilich erwiesen. ${ }^{2}$

Tralow, Johannes: Roxelane, a.a.O., S. 526.

2 Peirce, Leslie: The imperial harem: women and sovereignty in the Ottoman Empire. Oxford University Press, New York 1993. S. 291305 . 
Die Behauptung der türkischen Historiographie, die osmanischen Sklaven und Sklavinnen hätten ihre ethnische bzw. nationale Herkunft vergessen, ist ein Mythos der republikanischen Ära und hängt mit deren Assimilationsideologie und -politik zusammen. Es gibt ganz im Gegenteil viele historische Belege für ein aufrechterhaltenes ethnisches Bewusstsein und für eine entsprechende Parteilichkeit, die im Osmanischen Reich offensichtlich als normal galten. ${ }^{1}$

So entstand beispielsweise wegen Roxelanes polnischer Herkunft eine enge und aktive Diplomatie mit Polen. Dessen König, Sigismund I., konnte wahrscheinlich durch Roxelanes Einfluss den Frieden mit dem Osmanischen Reich aufrechterhalten. Roxelane korrespondierte häufig privat mit Sigismund und versicherte ihm, dass sie sich beim Sultan für seine Interessen einsetzen würde, wenn er sie ihr mitteile. Auch Roxelanes Tochter Mihrimah und ihr Schwiegersohn Rustem unterhielten einen regelmäßigen Briefwechsel mit dem polnischen König. ${ }^{2}$

1553 verlor Roxelane auch ihren zweiten Sohn Giangir. So hatte sie nur noch neben ihrer Tochter Mirmah ihren mittlerweile 22-jährigen, lebenslustigen und trunksüchtigen Sohn Selim und den 21-jährigen ehrgeizigen Sohn Bajazet, der äußerlich und charakterlich ganz nach seinem Vater ging. Die

${ }^{1}$ Coco, Carla: Sinnbild orientalischer Erotik. Stuttgart-Zürich, 1998, S. 89.

2 Peirce (1993), S. 294. 
restlichen Lebensjahre, die Roxelane noch zur Verfügung standen, versuchte sie zu nutzen, um die wachsende Rivalität zwischen diesen beiden Söhnen, den potentiellen Nachfolgern ihres Gatten, einzudämmen.

Als sie 1558 starb, blieb ihr immerhin erspart, miterleben $\mathrm{zu}$ müssen, wie sich der ehrgeizige und machtbesessene Bajazet 1559 in seinem Kampf gegen seinen Bruder Selim auch mit dem Vater überwarf und schließlich einen Aufstand gegen diesen selbst anzettelte. Als dieser fehlschlug, flüchtete er mit seinen vier Söhnen im November 1559 zum persischen Schah Tahmasp, der ihn erst wohlwollend aufnahm, aber letztendlich nach einer Zahlung Suleimans von 400000 Goldmünzen an dessen Bruder Selim übergab, der Bajazet und dessen sämtlichen Söhne 1561 erdrosseln ließ.

Das einzige Kind, das Suleiman außer Giangir nie enttäuscht hatte, war seine willensstarke Tochter Mirmah, die bei ihrem Vater mittlerweile die Rolle ihrer Mutter als seine Ratgeberin und Gesprächspartnerin eingenommen hatte.

Suleiman überlebte seine geliebte Roxelane um acht Jahre, bevor er auf seinem letzten Ungarnfeldzug 1566 starb. Für ihn gab es keine andere Frau mehr in seinem Leben. Er hielt seiner Gattin bis zum Tode die Treue. Neben ihr ließ er sich in der prächtigen Suleimaniye bestatten.

Zum ersten Mal in der Roxelane-Literatur zeichnet uns Tralow Roxelane, die durch Jahrhunderte als Vamp und Verbrecherin charakterisiert wurde, nicht als solche, sondern als Menschen und Frau, deren Handlungen der Dichter 
psychologisch zu erklären versuchte. In „Roxelane“ beschrieb Tralow den Einfluss von Roxelane auf Suleiman und deren Bedeutung in der osmanischen Geschichte wie den von Chadidscha auf den Propheten und deren Rolle zum Erscheinen des Islam.

„Ohnehin hatten sich das Reich und die Welt an das Dasein einer Roxelane Sultana längst gewöhnt. [...] Als sein „ehelich Gemahl, wie es Chadidscha war dem Propheten", hatte er um sie geworben. Mit Chadidscha, die als erste das Wort des Propheten, ihres Gatten, vernommen und sich mit ihm vor Allah geneigt hatte, war Roxelane von Suleiman verglichen worden, und wie Chadidscha die erste Frau des Islams, so sollte auch Roxelane die Erste, die Unvergleichliche sein. " ${ }^{\text {(1 }}$

Er nutzt die historische Konstellation aber auch für ein mildes politisches Plädoyer: Seine Roxelane ist, anders als der traditionelle europäische Blick es wollte, keine intrigante, machtgierige Hure, sie verkörpert Vernunft und Humanität in einer Welt, deren Orientierungsgrößen Staatsraison und absolutistische Willkür sind. ${ }^{2}$ So rechtfertigt Tralow ihre Verhalten und Haltungen gegenüber den Herausforderungen, die ihr begegneten.

\begin{abstract}
„Darf eine Mutter ihre Kinder nicht schützen? Ich will, dass sie leben! Ich bin eine Frau. Kann ich meine Söhne auf den Opferstein legen, wie es Abraham tat mit seinem Sohn Isaak? Will Gott das? Kann eine Frau das? Ich kann es nicht. ${ }^{\text {"3 }}$
\end{abstract}

\footnotetext{
${ }^{1}$ Tralow, Johannes: Roxelane, a.a.O., S. 483.

2 Vgl. Johannes Tralow 1882 - 1968 - Katharineum zu Lübeck, archive.is/HZtjT [stand: 9.6.2018].

3 Tralow, Johannes: Roxelane, a.a.O., S. 525.
} 
Zugleich erdichtet Tralow in der islamischen Geschichte durch die Veränderung der Figuren im Schlachtenvorfall. Denn er nannte Isaak anstatt von Ismā'il und dies steht im Widerspruch zu islamischer Geschichte

Wie in allen Romanen bringt Tralow seine Meinung zum Ausdruck:

„Der Westen“ ist keineswegs zivilisierter oder fortschrittlicher als die angreifenden Osmanen, sondern ein brutales Feudalkonglomerat im Zerfall. Ein weiteres Leitmotiv: die Klugheit der Frauen. ${ }^{1}$

Tralow deckt auch seiner Leserschaft eine Informationsfülle über eine vergessene Zeit und eine unbekannte Großzivilisation auf.

Man erlebt die vielfältigen Formen des orientalischen Hoflebens, seine Prachtentfaltung, seine Intrigen und seine Grausamkeiten - das alles ergibt ein farben- und detailreiches Bild des mittelalterlichen Orients.

Tralows Schreiben ist im Vergleich mit den ersten Romanen etwas konventioneller. Er tendiert zur Breite, gelegentlich auch etwas zum genretypischen Kitsch; aber langweilig wird es nicht. Die Sprache ist frisch, vorwärtsdrängend, expressives Stakkato, gelegentlich auch manieriert; die Szenen- und Perspektivwechsel schnell.

1 Vgl. Johannes Tralow 1882-1968:

(http://www.katharineum.de/rundgang/beruehmte/rundgang/beruehmte /johannes-tralow-1882-1968) [stand: 9.2.2018]. 


\section{Zusammenfassung und Schlussfolgerungen der Abeit}

Roxelane wird allgemein als Musterbeispiel für Macht und Einfluss einer Haremsdame angesehen.

Die unvergleichliche Stellung Roxelanes gründete allein im Wohlwollen des Sultans. Als erste Sklavenkonkubine in der Geschichte des Osmanischen Reiches machte ihr Sulaiman seine Haseki „die Lieblinge des Sultans“. Somit definierte sich Roxelanes Status über ihr gutes Verhältnis zum Sultan, und nicht wie zuvor über deren Mutterschaft. Erstmals seit Ende des 14. Jahrhunderts wurde die Rolle der Prinzenmutter nicht von derjenigen der Ehefrau getrennt, was in der osmanischen Gesellschaft auf Befremden stieß. Die emotionale wie auch physische Nähe zum Sultan eröffnete Möglichkeiten, welche keine andere Prinzenmutter in diesem Ausmaße besaß. Der Aufenthalt am Zentrum der Macht barg neben einer potentiellen Einflussnahme auf den Sultan und einem besseren Zugang zu Informationen nicht zuletzt auch mehr Gelegenheiten zur Bildung von politischen Netzwerken. ${ }^{1}$

Roxelane konnte die strengen Traditionen des Osmanischen Reiches überwinden. Sie hat erreicht, was niemand zuvor erreichen konnte:

- Roxelane war die erste Sklavinnenkonkubine, die von einem Sultan freigelassen und geheiratet wurde. Damit stieg sie in der Hierarchie der Sultansfrauen auf und übernahm nach dem Tod der Sultansmutter die führende Rolle unter den Frauen des Hofes.

${ }^{1}$ Vgl. Peirce (1993), S. 90. 
- Sie schenkte Suleiman mehrere Söhne, was im Widerspruch zum Prinzip ,ein Sohn pro Konkubine“ steht. ${ }^{1}$

- Nachdem sie aus dem Alten Serail, dem traditionellen Ort der weiblichen Angehörigen des Hofes, in das Topkapi-Serail umgezogen war und ihre Kinder als potentielle Sultansmutter dort erziehen lassen konnte, war sie dem Sultan so nahe wie bisher bestenfalls die Frauen der frühen osmanischen Herrscher.

- Roxelane war auch die erste Sultansfrau, die in der Hauptstadt blieb und ihre Söhne nicht in die Provinz begleitete, sondern dort nur besuchte. Ihre Anwesenheit in Istanbul trug dazu bei, dass sie immer mehr zur politischen Informantin, Mitwisserin und Beraterin des Sultans wurde. ${ }^{2}$ Die Briefe, die sie während seiner Feldzüge an den Sultan schrieb, enthielten politische Empfehlungen sowie wichtige Informationen über die Ereignisse in der Hauptstadt. Dies war für den Sultan von unschätzbarem Wert, denn die Feldzüge und seine langfristige Abwesenheit brachten die Gefahr mit sich, dass einer der Söhne den Thron an sich reißen konnte.

- Roxelane ermöglichte ihrer Tochter Mihrimah, deren Funktionen nach ihrem Tod zu übernehmen. Dazu gehörte Mihrimahs Verheiratung mit dem späteren Großwesir Rüstem Pascha.

${ }^{1}$ Kürsat (2003), S. 40.

${ }^{2}$ Ebenda, S. 40. 
- Sie war die erste Frau im Haushalt eines Sultans, die ihre Bedeutung durch den Bau einer Freitagsmoschee samt Külliye im alten Istanbul sichtbar machte.

Die nun auch räumliche Nähe zum Sultan und dem politischen Zentrum bedeutete eine neue Machtquelle. Der beständige Aufenthalt Roxelanes in der Hauptstadt machte sie zu einer wichtigen Verbündeten des Sultans. ${ }^{1}$

Roxelane wurde als Erfolgsmodell für die diplomatische Beteiligung der Frau zur Zeit Suleimans betrachtet, wie die enge und aktive Diplomatie mit Polen. Dessen König, Sigismund I., konnte wahrscheinlich durch Roxelanes Einfluss den Frieden mit dem Osmanischen Reich aufrechterhalten.

Tralows Sympathie mit Roxelane und ihre Beschreibung als Menschen und Frau - nicht als Vamp und Verbrecherin wie sie in den europäischen Literaturen charakterisiert wurde -, deren Handlungen er psychologisch zu erklären versuchte.

Durch diese konkrete historische Bestimmtheit des epischen Geschehens gelingt Tralow, einen vollgültig historischen Roman zu schaffen. Der Roman zeigt in vielen Bildern Roxelane als kluge Frau, die die strengen Gesetze des osmanischen Reiches überwinden konnte und das Recht hat, sich für ihre Kinder vor dem Mord einzusetzen.

\footnotetext{
${ }^{1}$ Vgl. Schweisgut, Susanne: Macht und Einfluss der Frau am Hofe Karls V. und Süleymans I. Ein Vergleich, SE-Arbeit, SS 2010, S. 435.
} 


\section{Literaturverzeichnis}

\section{Deutsche Primär- und Sekundärliteratur:}

- Abu-Hattab, Muhammad: Zum Bild des Propheten Muhammad in Bezug auf seine Darstellung in Europa. Betrachtungen eines arabischen Germanisten. In: Wissenschaftliche Zeitschrift der Fremdsprachenfakultät an der Al-Azhar Universität, 6/1982.

- Andrews, Walter: Poetry's Voice, Society's. 1985, Seattle

- Barber, Noel: Die Sultane - Die Geschichte des Othmanischen Reiches dargestellt in Lebensbildern, Frankfurt a.M., Berlin, Wien $1975^{2}$.

- Brandt, Bruno: Ein Leben für den historischen Roman, In: Börsenblatt für den deutschen Buchhandel, Leipzig 134/1967.

- Deutsches Literatur-Lexikon, begründet von Wilhelm Kosch u.a., Bnd. 23, K.G. Sauer Verlag, Zürich und München.

- Hammer-Purgstall (Freiherr von), Joseph, Geschichte des osmanischen Reiches, Bd. 3: Vom Regierungsantritte Suleiman des Ersten bis zum Tode Selim's II. 1520-1574, Pest 1828.

- Johannes Tralow, Der Nachlass, Hrsg. H. Stötzer, H. Döhn, Berlin 1977, Deutsche Staatsbibliothek

- Kaufmann, Max R.: Osmanische Trilogie. Die historischen Romane von Johannes Tralow. In: Mitteilungen der Deutsch-Türkischen Gesellschaft e.v. Bonn, Nr. 10 vom April 1956.

- Kürsat, Elçin: Haremsfrauen und Herrschaft im Osmanischen Reich. In: Zeitschrift: Nachdenken über..., Band: 21, Heft: 1, Stuttgart, 2003. 
- Lamb, Harold: Suleiman der Prächtige, München 1952.

- Lexikon der deutschsprachigen Gegenwartsliteratur, begründet von Herrmann Kunisch u.a., Nymphenburger Verlagshandlung.

- Marchlewitz, Ingrid: Irmgard Keun: Leben und Werk, Königshausen und Neumann Verlag, Würzburg, 1999.

- Martini, Fritz: Deutsche Literaturgeschichte von den Anfängen bis zur Gegenwart, Alfred Kröner Verlag, Stuttgart, 1958. 9. Auflage.

- Meram, Ali Kemal: Padisah Analari. Istanbul, 1997.

- Meyers Neues Lexikon, 2. Aufl., Leipzig 1976, Bd. 13.

- Peirce, Leslie, The Imperial Harem. Women and Sovereignty in the Ottoman Empire (Studies in the Middle Eastern history), Oxford-New York-Toronto u.a.1993.

- Penzhold, Leonhard: Tralow-Ausstellung der Deutschen Staatsbibliothek, In: Der Bibliothekar, Leipzig 21/1967.

- R G H: Johannes Tralow, ein Meister des historischen Romans, In: Mittelungen der Deutsch-Türkischen Gesellschaft, Bonn 76/1967.

- Schreiber, Hermann(Hrsg): Weltreiche(2)- Das Imperium Romanum. Das Heilige Römische Reich. Byzanz. Die Osmanen.

- Schweisgut, Susanne: Macht und Einfluss der Frau am Hofe Karls V. und Süleymans I. Ein Vergleich, SEArbeit, SS 2010.

- Sternheim, Carl: „Vermischte Schriften“, Deutschlands Regisseure. In: Tage-Buch. Berlin 23/1922.

- Tieze, Wilhelm: „Pg. Vor der Spruchkammer. Porträt eines deutschen Schriftstellers.“ In: Der Ruf. 
Unabhängige Blätter der jungen Generation. München 9/1946.

- Tralow, Johannes: Roxelane, Verlag der Nation, 13. Auflage, Husum, 2008.

- Tralow, Johannes: Leben und Werk, (Hg) Deutsche Staatsbibliothek, Berlin. 1968.

- Wildermanns, Hanns: Osmanische Tetralogie-Zum 85. Geburtstag des Schriftstellers Johannes Tralow am 2.8.1967, In: Mitteilungen der Deutsch-Türkischen Gesellschaft, Bonn 70/1967.

$$
\text { - المر اجع العربية }
$$

\section{Internetwebsite:}

- Iqpal, Muhammad: Enzyklopädie des Islam, (http://www.eslam.de/begriffe/i/iqbal.htm) veröffentlicht im Jahre 2006.

- Mehmed II.: Wikipedia, (http://de.wikipedia.org/wiki/Mehmed_II.) veröffentlicht im Jahre 2003.

- Johannes

Tralow

1882-1968: (http://www.katharineum.de/rundgang/beruehmte/rundga ng/beruehmte/johannes-tralow-1882-1968) 2011 